\title{
Community-Based Management and Treatment Services for Psychosis - China, 2019
}

\author{
Miaomiao Zhao'; Ning Ma ${ }^{1, *}$; Xun Wang'; Xiamin Wu'; Wufang Zhang'; Runzi Chen ${ }^{1}$; \\ Yulu Wang'; Lili Guan'; Hong $\mathrm{Ma}^{1}$; Xin $\mathrm{Yu}^{1}$; $\mathrm{Lin}^{1} \mathrm{Lu}^{1}$
}

\begin{abstract}
Introduction: Community-based services for psychosis are an important part of mental health services in China. We analyzed community-based follow-up and treatment services for psychosis in China in 2019 to provide evidence for policymaking and services delivering.

Methods: Data from the National Information System for Psychosis were used to analyze usage of the information system and the registration, management, and treatment situation of patients with psychosis in China in 2019 and compared the results of 2019 with that of 2018.

Results: In 2019, $100 \%$ of cities and counties used the information system and 6,230,157 patients were registered. In 2019, there were 5,944,724 registered patients $(94.05 \%)$ in China under community-based follow-up services and 5,375,252 patients $(85.04 \%)$ had regular follow-ups; 5,295,657 patients (83.78\%) were treated by taking antipsychotics and 3,354,251 $(53.07 \%)$ patients took medication regularly; $4,974,314$ patients at home $(87.81 \%)$ were in stable condition; and there was a significant difference in patients' stable condition and medication-taking rates among eastern, central, and western regions $(p<0.05)$. Compared with 2018, the nationwide regular management rate, medication-taking rate, regular medication-taking rate, and patients' stable rate increased by $2.35 \%, 2.48 \%, 11.29 \%$, and $7.21 \%$ respectively.

Conclusions and Implications for Public Health Practice: Compared with 2018, the level of management and treatment improved significantly but still needs to be further strengthened, especially in western China.
\end{abstract}

\section{INTRODUCTION}

Psychosis is early onset, long course, easily recurrent, highly disabling, and has a heavy disease burden (1). Most patients with psychosis need to take long-term medication to control the disease. Over the past decade, the Chinese government committed to developing community-based follow-up and treatment services for psychosis, which is an important part of mental health services in China. In 2009, the community-based follow-up services of patients with 6 kinds of psychoses* were included in the national basic public health services (2). In this study, we analyzed community-based follow-up and treatment of patients with psychosis in China in 2019 and compared the results with indicators from 2018 to explore progress, discussed key issues in communitybased service delivery, and provided references for the government on policymaking and service delivery in mental health.

\section{METHODS}

Data from the National Information System for Psychosis of China was used, which contained information on demographic characteristics, mental state, and follow-up and treatment records in community with six confirmed types of psychoses. Psychiatrists were responsible for clarifying diagnoses, inputting the data, and transferring patients' information to local communities through the system. Community mental health workers were responsible for the screening and follow-up of patients with psychosis in the community and inputting relevant data promptly into the information system. Workers in health administrative departments and mental illness prevention and control technology management institutions were responsible for auditing and quality control of data. The number of permanent residents was derived from data of China CDC as of December

\footnotetext{
* Psychoses as defined by National Basic Public Health Services include schizophrenia, delusional disorders, schizoaffective disorder, bipolar disorder, psychotic disorder due to epilepsy, and mental retardation with psychotic symptoms. Psychosis in this study referred to the six kinds of psychoses.
} 


\section{1, 2019.}

SPSS 16.0 (SPSS Inc.) was used for data cleaning and analysis. Descriptive analysis and Kruskal-Wallis Test were used to analyze usage of the information system and the quality of the data on registration and community-based follow-up and treatment of patients with psychosis in China and its regions (the duration of service provision was from January 1, 2019 to December 31, 2019) and to compare the results of 2019 with 2018. The analytical unit of correlation analysis and regional differences in the analysis were at the provincial level. The statistical significance level $(\alpha)$ was set at 0.05 .

According to the routine work requirements in community-based follow-up and treatment services for psychosis, 6 indicators were selected as the main statistical indicators in the study: 1) registration rate $(\%)=$ the number of registered patients/the total number of permanent residents in the jurisdiction $x$ $100 \%$; registered patients refer to patients who were alive by December 31, 2019; 2) management rate $(\%)=$ the number of patients under follow-up services/the number of registered patients $\times 100 \%$; patients under follow-up services referred to patients with at least one complete follow-up on record in 2019 ; 3) regular management rate $(\%)=$ the number of patients under regular follow-up services/the number of registered patients $\times 100 \%$; patients under regular follow-up services referred to patients who were followed up at least once every three months in 2019; 4) medication-taking rate $(\%)=$ the number of patients taking antipsychotics/the number of registered patients $\times 100 \%$; patients taking antipsychotics referred to patients who had at least one follow-up record of taking antipsychotics in 2019; 5) regular medication-taking rate $(\%)=$ the number of patients taking antipsychotics regularly/the number of registered patients $\times 100 \%$; patients taking antipsychotics regularly referred to patients taking medication according to the doctor's prescription in 2019 ; 6) patients' stable rate $(\%)=$ the number of patients with stable condition of disease/the number of patients at home under follow-up services $\times 100 \%$; patients with stable condition of disease referred to patients who were in stable condition in each followup record in 2019.

\section{RESULTS}

In 2019, 333 cities and 2,854 districts/counties across the country all used the National Information
System for Psychosis. This was the first year to achieve full coverage of all cities and counties in the information system (332 cities and 2,832 counties were covered in 2018).

There were 6,230,157 patients with psychosis registered in the information system on December 31, 2019 (236,103 more cases, a $3.94 \%$ increase, compared with that on December 31, 2018). Among registered patients, schizophrenic patients accounted for $72.09 \%$; males accounted for $50.86 \%$; working-age patients (18-59 years old) accounted for $74.57 \%$; married patients accounted for $54.08 \%$; $67.45 \%$ of patients lived in rural areas; $59.49 \%$ of patients were farmers and only $2.60 \%$ were employed; $80.97 \%$ of patients' education level were with junior high school or below. (Table 1)

In 2019, the national registration rate of psychosis was $0.449 \%$, with $3.46 \%$ increase compared with 2018 $(0.434 \%)$. Among all 31 provincial-level administrative divisions (PLADs) and Xinjiang Production and Construction Corps, the registration rate in Hubei Province was the highest $(0.501 \%)$. (Table 2 )

By the end of 2019, 5,944,724 patients in China were under community-based follow-up services with an annual management rate of $94.05 \%$, which was slightly lower than that of 2018 (94.90\%). A total of $5,375,252$ patients were under regular follow-up services with an annual regular management rate of $85.04 \%$, which was $2.35 \%$ higher than that of 2018 (82.69\%). Among all 31 PLADs and Xinjiang Production and Construction Corps, Hunan Province had the highest management rate (97.81\%), and Shanghai Municipality had the highest regular management rate $(92.68 \%)$. (Table 2 )

Moreover, 5,295,657 patients were treated by taking antipsychotics in 2019 with an annual medicationtaking rate of $83.78 \%$, which was $2.48 \%$ higher than that of $2018(81.30 \%)$. A total of 3,354,251 patients took antipsychotics regularly with an annual regular medication-taking rate of $53.07 \%$, which was $11.29 \%$ higher than that of 2018 (41.78\%). In 2019, there were $4,974,314$ patients in stable condition, with an annual patients' stable rate of $87.81 \%$, which was $7.21 \%$ higher than that of $2018(80.60 \%)$. Among all 31 PLADs and Xinjiang Production and Construction Corps, Hunan Province had the highest medicationtaking rate $(94.66 \%)$, Beijing Municipality had the highest regular medication-taking rate $(75.21 \%)$, and Shanghai Municipality had the highest patients' stable rate $(98.03 \%)$. (Table 2$)$

In 2019, significant differences existed among 
TABLE 1. Demographic characteristics and diagnosis of real-time registered patients with psychosis - China, 2019.

\begin{tabular}{|c|c|c|}
\hline Characteristics & Number of patients & Constituent ratio $(\%)$ \\
\hline \multicolumn{3}{|l|}{ Gender } \\
\hline Male & $3,168,381$ & 50.86 \\
\hline Female & $3,061,776$ & 49.14 \\
\hline \multicolumn{3}{|l|}{ Age, years } \\
\hline$<18$ & 111,552 & 1.79 \\
\hline $18-44$ & $2,399,698$ & 38.52 \\
\hline $45-59$ & $2,246,142$ & 36.05 \\
\hline$\geq 60$ & $1,472,765$ & 23.64 \\
\hline \multicolumn{3}{|l|}{ Residential area type } \\
\hline Urban & $1,882,938$ & 30.22 \\
\hline Rural & $4,202,474$ & 67.45 \\
\hline Unknown & 144,745 & 2.32 \\
\hline \multicolumn{3}{|l|}{ Educational level } \\
\hline Illiterate or semi-illiterate & $1,641,161$ & 26.34 \\
\hline Primary school or junior high school & $3,403,397$ & 54.63 \\
\hline Senior high school or technical secondary school & 591,346 & 9.49 \\
\hline College and above & 323,332 & 5.19 \\
\hline Unknown & 270,921 & 4.35 \\
\hline \multicolumn{3}{|l|}{ Ethnicity } \\
\hline Han & $5,858,214$ & 94.03 \\
\hline Minority & 371,943 & 5.97 \\
\hline \multicolumn{3}{|l|}{ Marriage } \\
\hline Unmarried & $2,236,031$ & 35.89 \\
\hline Married & $3,369,046$ & 54.08 \\
\hline Widowed & 201,051 & 3.23 \\
\hline Divorced & 296,366 & 4.76 \\
\hline Unknown & 127,663 & 2.05 \\
\hline \multicolumn{3}{|l|}{ Occupation } \\
\hline Employed & 162,246 & 2.60 \\
\hline Farmers & $3,706,379$ & 59.49 \\
\hline Laid off or unemployed & 740,243 & 11.88 \\
\hline Students & 218,851 & 3.51 \\
\hline Retired & 172,534 & 2.77 \\
\hline Other & $1,229,904$ & 19.74 \\
\hline \multicolumn{3}{|l|}{ Diagnosis } \\
\hline Schizophrenia & $4,491,447$ & 72.09 \\
\hline Delusional disorders & 28,306 & 0.45 \\
\hline Schizoaffective disorder & 91,543 & 1.47 \\
\hline Bipolar disorder & 423,654 & 6.80 \\
\hline Psychotic disorder due to epilepsy & 344,630 & 5.53 \\
\hline Mental retardation with psychotic symptoms & 850,577 & 13.65 \\
\hline In total & $6,230,157$ & 100.00 \\
\hline
\end{tabular}




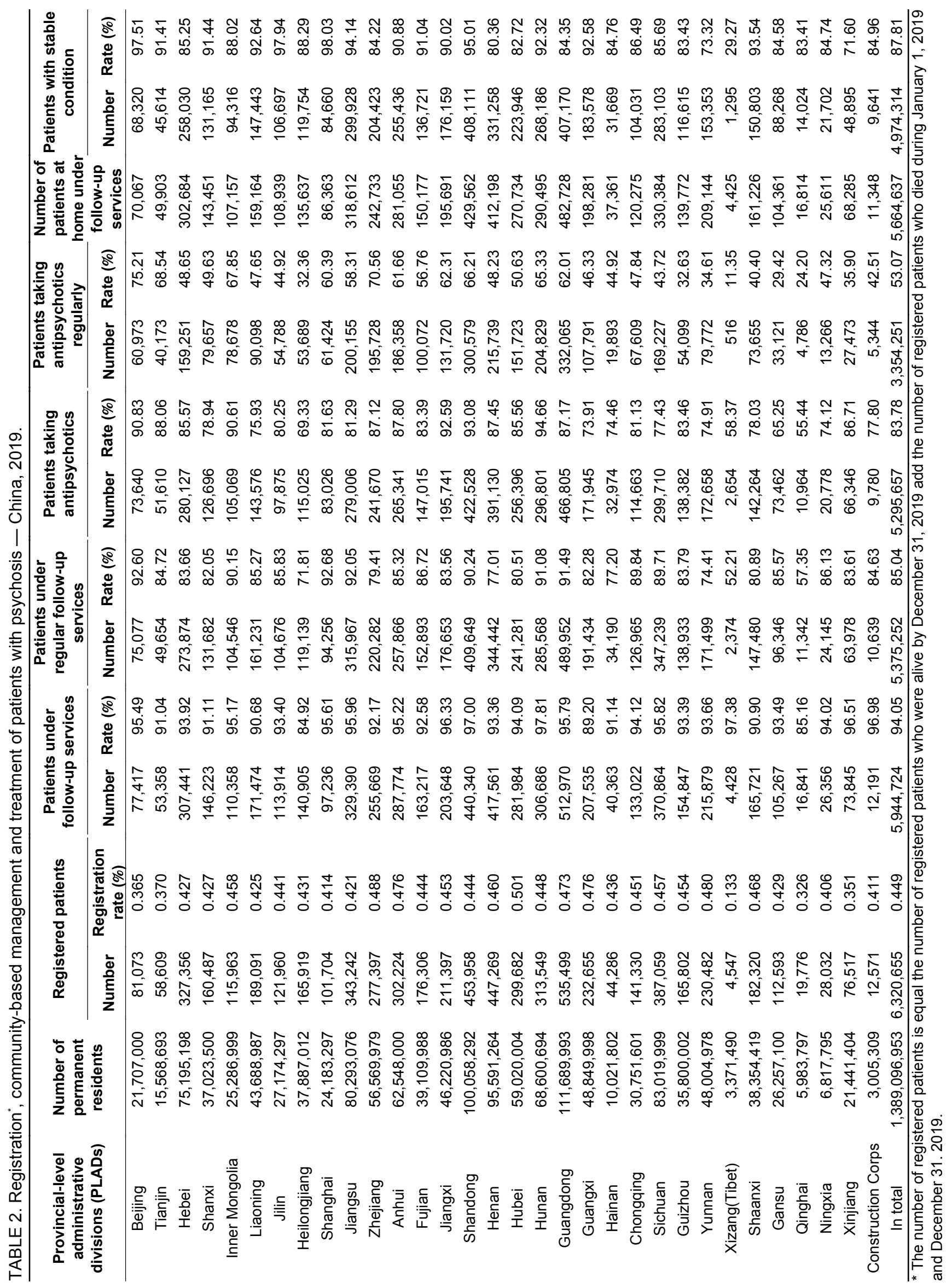


eastern, central, and western regions of $\mathrm{China}^{\dagger}$ in patients' medication-taking rate, regular medicationtaking rate, and stable rate $(p<0.05)$. All the three rates were the lowest in the western region. (Table 3 )

\section{DISCUSSION}

The Comprehensive Mental Health Action Plan 2013-2020 adopted in the $66^{\text {th }}$ World Health Assembly made 2 of the 4 objectives focused on providing comprehensive, integrated, and responsive mental health and social care services in communitybased settings and strengthening information systems, evidence, and research for mental health (3). In recent years, the Chinese government has put the two objectives as priority areas in enhancing the mental health service system and issued a series of policy projects, such as National Mental Health Work Plan (2015-2020) (4) and National Comprehensive Management Pilot Project for Mental Health Service (5), and built the National Information System for Psychosis in 2011. The coverage of community-based follow-up and treatment services for psychosis in China has been expanding year over year for both coverage areas and registered patients, and the registration rate has increased by $41.6 \%$ in 5 years (6-9).

The overall level of community-based follow-up and treatment services for psychosis has been improved in 2019. In past 5 years, the nationwide management rate, regular management rate, medication-taking rate, regular medication-taking rate and patients' stable rate increased by $27.56 \%, 137.74 \%, 139.10 \%, 196.15 \%$, and $29.82 \%$ respectively (6). The National Mental Health Work Plan of China (2015-2020) (4) laid out 5-year work objectives on community-based management and treatment for psychosis and has contributed greatly to the rapid improvement. Since the work plan launched in 2015, the National Health Commission (NHC) of China has actively issued relevant policies (10) and work regulations (11), carried out national pilot projects on comprehensive management for mental health jointly with multiple departments (5), conducted nationwide capacitybuilding training especially in the western region, and organized work experience exchange among PLADs and pilot cities. In the meanwhile, provincial and municipal governments issued a number of healthcare security and life assistance policies, raised funds on mental health through multiple channels and conducted technical guidance and personnel training especially for practitioners at the county level and below to enhance their ability to diagnose and treat mental illness. To a considerable extent, these measures promoted the improvement of the provision and quality of community-based services for psychosis in the last 5 years.

In addition, the rates of medication-taking have improved significantly between 2019 and 2018. The gap between patients' medication-taking rate and regular medication-taking rate was $30.71 \%$ in 2019 . Although the gap is smaller than that of 2018 (39.52\%), it is still needed to be further reduced through enhancing healthcare security policies and more health education to improve patients' medication compliance. Similarly, the regular management rate was $9.01 \%$ lower than the management rate in 2019, which revealed that quality control of follow-up services needed to be further strengthened.

Regional differences in patients' medication-taking and stable condition were also significant in 2019 . PLADs with better comprehensive services performance were mainly concentrated in the eastern region and were interspersed in the central region.

TABLE 3. Regional comparison of community-based follow-up and treatment services for psychosis - China, 2019.

\begin{tabular}{|c|c|c|c|c|c|}
\hline & Eastern Region $(\mathrm{N}=11)$ & Central Region $(\mathrm{N}=8)$ & Western Region $(\mathrm{N}=13)$ & $H$ & $p$ \\
\hline Registration rate $(\%)$ & 0.441 & 0.459 & 0.449 & 2.511 & 0.285 \\
\hline Management rate $(\%)$ & 94.61 & 93.88 & 93.42 & 0.046 & 0.977 \\
\hline Regular management rate $(\%)$ & 87.97 & 82.14 & 84.05 & 4.424 & 0.109 \\
\hline Medication-taking rate $(\%)$ & 85.85 & 86.28 & 77.70 & 7.749 & 0.021 \\
\hline Regular medication-taking rate $(\%)$ & 60.27 & 53.31 & 41.82 & 13.519 & 0.001 \\
\hline Patients' stable rate $(\%)$ & 89.82 & 87.73 & 84.81 & 6.042 & 0.049 \\
\hline
\end{tabular}

\footnotetext{
$\dagger$ Eastern region consists of 11 provincial-level administrative divisions (PLADs) (Beijing, Tianjin, Hebei, Liaoning, Shanghai, Jiangsu, Zhejiang, Shandong, Fujian, Guangdong, and Hainan); central region consists of 8 PLADs (Shanxi, Jilin, Heilongjiang, Anhui, Jiangxi, Henan, Hubei, and Hunan); western region consists of 12 PLADs (Inner Mongolia, Guangxi, Chongqing, Sichuan, Guizhou, Yunnan, Xizang (Tibet), Shaanxi, Gansu, Qinghai, Ningxia, and Xinjiang) and Xinjiang Production and Construction Corps.
} 
Community-based services in the western region needed to be further improved, especially in patients' medication-taking. The government should investigate and analyze difficulties in community-based mental health services in the western region, further focus on priority areas, and take effective measures such as providing financial support, more practical capacity building training, and appropriate skills guidance to remove barriers in service delivery and improve patients' stable condition in the western region.

In conclusion, patients' stable condition and community-based services provision for psychosis in 2019 were better than those in 2018, and all work indicators have been significantly improved in the past 5 years. In the future, more attention should be paid on improving quality of services (e.g. medicationtaking) and strengthening community-based services delivery in the western region.

Acknowledgments: Special thanks to workers in health institutions at all levels for collecting and reporting patients' information in accordance with relevant requirements and regulations of the government and to workers in health administrative departments and mental illness prevention and control technology management institutions at all levels for the audit and quality control of the data.

Conflicts of interest: No conflicts of interest were reported.

\section{doi: $10.46234 / \mathrm{ccdcw} 2020.217$}

\#Corresponding author: Ning Ma, maning@bjmu.edu.cn.

\footnotetext{
${ }^{1}$ Peking University Sixth Hospital, Peking University Institute of Mental Health, NHC Key Laboratory of Mental Health (Peking University), National Clinical Research Center for Mental Disorders (Peking University Sixth Hospital), Beijing, China.
}

Submitted: September 21, 2020; Accepted: October 03, 2020

\section{REFERENCES}

1. Huang YQ, Wang Y, Wang H, Liu ZR, Yu X, Yan J, et al. Prevalence of mental disorders in China: a cross-sectional epidemiological study. Lancet Psychiatry 2019;6(3):211 - 24. http://dx.doi.org/10.1016/ S2215-0366(18)30511-X.

2. Ministry of Health of the People's Republic of China. National basic public health services regulates (2009). 2009. http://www.nhc.gov. cn/jws/s3581r/200910/fe1cdd87dcfa4622abca696c712d77e8.shtml?fro $\mathrm{m}=$ singlemessage. [2020-9-10]. (In Chinese).

3. World Health Organization. Comprehensive mental health action plan 2013-2020. 2013. https://www.who.int/mental_health/action_plan_ 2013/en/. [2020-9-10].

4. General Office of the State Council. National mental health work plan of the People's Republic of China (2015-2020). 2015. http://www.gov. cn/zhengce/content/2015-06/18/content_9860.htm. [2020-9-10]. (In Chinese).

5. Zhang WF, Ma N. China's national comprehensive management pilot project for mental health. BJPsych Int 2017;14(2):44-6. http://dx.doi.org/10.1192/s2056474000001781.

6. Wang X, Ma N, Wang LY, Yan J, Jin TL, Wu XM, et al. Management and services for psychosis in People's Republic of China in 2014. Chin J Psychiatry 2016;49(3):182 - 8. http://dx.doi.org/10.3760/cma.j.issn. 1006-7884.2016.03.012. (In Chinese).

7. Wu XM, Ma N, Wang LY, Zhang SB, Wang X, Zhang WF, et al. Management and services for psychosis in People's Republic of China in 2015. Chin J Psychiatry 2017;50(4):302 - 7. http://dx.doi.org/10.3760/ cma.j.issn.1006-7884.2017.04.013. (In Chinese).

8. Wang X, Ma N, Wang LY, Zhang SB, Wu XM, Zhang WF, et al. Management and services for psychosis in People's Republic of China in 2016. Chin J Psychiatry 2018;51(1):47 - 52. http://dx.doi.org/10.3760/ cma.j.issn.1006-7884.2018.01.010. (In Chinese).

9. Wu XM, Ma N, Wang X, Zhang WF, Guan LL, Ma H, et al. Management and services for psychosis in People's Republic of China in 2017. Chin J Psychiatry 2019;52(1):82 - 8. http://dx.doi.org/10.3760/ cma.j.issn. 1006-7884.2019.01.008. (In Chinese).

10. Chen RZ, Wu XM, Ma N. Mental health policy and implementation from 2009 to 2019 in China. Chin Ment Health J 2020;34(7): 555 60. http://zxws.cbpt.cnki.net/WKD/WebPublication/paperDigest.aspx? paperID=5adc5542-758b-4ed9-b8ce-2c3132431d8b. (In Chinese).

11. National Health Commission of the People's Republic of China. Work regulation on management and treatment for psychosis (2018). 2018. http://www.nhc.gov.cn/jkj/s7932/201806/90d5fe3b7f48453db9b9beb 85dfdc8a8.shtml. [2020-9-10]. (In Chinese). 